\title{
Applications of transgenic technology in ornamental fish
}

\author{
ZHIXUAN GONG, HAIYAN WAN, MINGRU CHEN, BENSHENG JU, and TIE YAN
}

Department of Biological Sciences, National University of Singapore, Singapore (Email: dbsgzy@inus.edu.sg)

SUMMARY: The transgenic technology has been widely used in biotechnology, from generation of genetically modified (GM) foods to production of pharmaceutical proteins. Although fast-growing transgenic fish using exogeneous growth hormone gene have been successfully produced, the marketing of such GM food fish remains to face an uphill battle. In the present study, we attempted to use the transgenic technology to develop novel varieties of ornamental fish. So far, we have succeeded in generation of several colourful transgenic zebrafish by transferring a jellyfish green fluorescent protein ( $g f p)$ gene and an anemone red fluorescent protein ( $f p)$ gene. These transgenic zebrafish displayed green, red, yellow or orange fluorescent colors under both daylight and uv light. Thus, our work demonstrated the feasibility to generate novel varieties of ornamental fish by the transgenic techniques. Currently we are conducting mating behaviour experiments using these transgenic zebrafish. We are also aiming at development of biomonitoring transgenic fish by using some inducible gene promoters that can respond certain environmental pollutants.

KEY WORDS: BFP, CFP, dsRed, elastase, epidermis, EST, fluorescent, GFP, keratin, muscle, myosin light chain, pancreas, skin, RFP, YFP

\section{INTRODUCTION}

The transgenic technique has been applied to the zebrafish for more than a decade. ${ }^{1)}$ However, the pace of transgenic zebrafish is rather slow at the beginning because of the lack of zebrafish gene promoters for analysis. As a results, essentially all early transgenic zebrafish works employed gene promoters from non-fish species. ${ }^{2,3,4}$ ) In recent years, following the successful demonstration of the faithful expression of transgenic GFP under a zebrafish gatal promoter, ${ }^{5)}$ increasing number of transgenic zebrafish works has been reported using zebrafish gene promoters, including those from $\alpha$ actin and $\beta$-actin, ${ }^{6)}$ islet $1,{ }^{7)}$ hsp $70,{ }^{8)} H u c,{ }^{9)} \beta$ tubulin $^{10)}$ and robopsin. ${ }^{11)}$ In all of these works, the gfp gene isolated from a species of jellyfish, Aequarie Victoria, ${ }^{12)}$ was used to express in transgenic zebrafish under several zebrafish promoters and dynamic patterns of GFP expression were observed in a non-invasive way. In all cases the transgenic expression mimics the expression of the endogenous genes from which the promoters were derived.

In the present communication, we will briefly summerize our progress in generation of $g f p$ transgenic zebrafish under several different tissuespecific promoter isolated from zebrafish and discuss the potential applications of these $g f p$ transgenic zebrafish.

\section{SELECTION OF TISSUE-SPECIFIC ZEBRAFISH CDNA CLONES}

Our transgenic work was originated from our EST (express sequence tag) project. In the past few years, our laboratory has generated about 3,000 zebrafish EST clones from several zebrafish cDNA libraries, mainly from an embryonic library, an adult library, a testis library and an ovary library. ${ }^{13,14)}$ About half of these clones can be identified with confidence by sequence homology search. From our collection, we have found tissue-specific clones representing essentially all major tissues and organs. The summary of representative tissue-specific and inducible cDNA clones from our EST collection is shown in Table 1. The tissue specificity of expression of many of these clones have been confirmed by northern blot analysis and in situ hybridization.

\section{ISOLATION OF ZEBRAFISH GENE PROMOTERS}

Selected tissue-specific cDNA clone were used to isolate their promoter regions. In our laboratory, in general, two methods have been used to isolate the promoters. One is linker-mediated PCR and the other is PCR navigated genomic library screening. The former has been described in detail in our previous publication. ${ }^{15)}$ Here we just describe the 
second method. Briefly, an amplified genomic library (usually with $>2 \times 10^{6}$ primary clones) was first subdivided into over 20 sublibraries, each of which has about 70,000 clones. A pair of PCR primers was synthesized based on 5' sequence of a cDNA clone and used for PCR screening. A positive sublibrary would be further divided with fewer clones and screened by PCR. The subdivision approach would be repeated until a PCR-positive sublibrary is small enough (usually $<100$ pfu per plate) to apply PCR to individual clones for identification of a single positive clone.

Table 1 Example of tissue-specific and inducible zebrafish cDNA clones

\begin{tabular}{ll}
\hline $\begin{array}{l}\text { Tissue } \\
\text { specificity/inducibility }\end{array}$ & Encoded proteins \\
\hline Cental nervous system & Vimentin, AGFP \\
Eyes & Crystallins \\
Heart & Cardiac $\alpha$-actin \\
Erythrocytes & Globins \\
Liver & Transferrrin etc. \\
Pancreas & Elastases, Trypsin etc. \\
Intestine & Fatty acid binding \\
& protein etc. \\
Kidney & Nephrosin \\
Ovary & ZP2, ZP3 etc. \\
Testis & Testicular tectin \\
Skeletal muscle & MLC2f (Mylz2), CKM \\
Skin & Kertins etc. \\
Estrogen-induced & Vitellogenins \\
Heat shock/heavy metal & Hsp25, Hsp70, Hsp91 etc \\
induced & \\
Ubiquitous & EF1 $\alpha$, ribosomal proteins \\
\hline
\end{tabular}

By the two methods, we have so far isolated six different zebrafish gene promoters. These promoters displayed various tissue specificity such as in skin (keratin8 or krt8), muscle (creatine kinase muscle or $\mathrm{ckm}$, and myosin light polypeptide 2 or mylz2) and pancreas (elastase $A$ or ela $A$ ). Some of them have potential inducibility such as by heavy metals (heat shock protein 25 or hsp25) or by estrogenic compounds (vitellogenin or $\mathrm{vg}$ ). One of them (acidic ribosomal phosphaproterin $P 0$ or arp) functions ubiquitously. All of these promoters have been linked with the $g f p$ reporter gene. Some of them were also linked with a recently isolated $r f p$ reporter gene. ${ }^{16)}$

\section{GENERATION OF LIVING COLOR TRANSGENIC ZEBRAFISH}

Previously, by transient expression in zebrafish embryos and fry, we have domenstrated the faithful expression of three of these promoters: krts (previously named $c k$ for cytokeratin), ckm (previously named $m c k$ for muscle creatine kinase) and $a r p .{ }^{17)}$ The two muscle specific promoters, mylz2 (previously named $M L C 2 f$ for myosin light chain 2, fast muscle isoform) and ckm, were Ialso tested in adult fish by direct injection of naked DNA into trunk muscles in the zebrafish. ${ }^{18)}$ Recently, transgenic works using the mylz2, elaA, vtg and $h s p 25$ promoters were also initiated or carried out.

In general, successful generation of transgenic fish can only be claimed after stable transgenic lines are established. Usually, the founder transgenic fish are mosaic and thus variable transmission rate from $<10 \%$ to $>50 \%$ can be observed in $\mathrm{F} 1$ offspring. If a stable line is obtained, F2 offspring from transgenic F1 should follow standard Mendelian ratios; i.e. when a heterologous (or hemizygous) transgenic F1 is crossed with a wild type fish, about $50 \%$ of F2 offspring should is transgenic. Based on this rule, we have obtained stable $g f p$ transgenic lines for three of our transgenic DNA constructs: krt8-gfp, $m y l z 2-g f p$ and elaA-gfp. In addition, we have also generated a stable transgenic line by insertion of two transgenic DNA constructs: $k r t 8-g f p$ and mylz2-rfp.

\section{A. $k r t 8-g f p$ transgenic zebrafish}

We have obtained five stable transgenic zebrafish lines with the chimeric construct, $k r t 8-g f p$. In all examined transgenic lines, GFP is expressed specifically in the outmost layer of skin epidermis and the innermost layer of epithelia in pharynx, esophagus and foregut, but not in the mid-gut and hind-gut. This pattern of expression is essentially identical to that of the endogenous $k r t 8$ expression. Thus, the exogenous $k r t 8$ promoter is faithfully activated in the transgenic zebrafish system.

\section{B. mylz2-gfp transgenic zebrafish}

$m y l z 2$ is a muscle specific gene and it is specifically expressed in fast skeletal muscles. ${ }^{19)}$ Two lines of mylz2-gfp transgenic zebrafish have been generated. In both lines, GFP expression is restricted to fast skeletal muscles but not to the slow muscles, which are located in the superficial layer of myotomes and can be distinguished by staining with a monoclonal antibody, F59, specifically against a slow myosin isoform. ${ }^{20)}$ After $48 \mathrm{hpf}$, GFP expression was also observed in head muscles including eye, jaw and gill muscles. This pattern of expression is identical 
to that of endogenous mylz 2 expression. ${ }^{18}$ Thus, this work again demonstrated the faithful expression mylz2 promoter in transgenic zebrafsih. Moreover, in both transgenic lines, GFP expression is so strong that green fluorescence can be easily observed from transgenic zebrafish even under a normal daylight. This observation provides a basis for generation of fluorescent ornamental fish as new varieties using the $g f p$ reporter gene.

\section{C. elaA-gfp transgenic zebrafish}

Elastase A is synthesized and secreted from exocrine cells in pancreas. The transgenic zebrafish containing elaA-gfp express GFP specifically in exocrine cells but not in endocrine cells of the pancreas, indicating the high specificity of the ela $\mathrm{A}$ promoter. This transgenic fish line will be useful to trace pancreas development and to purify pancreas cells for in vitro culture and for establishment of cell type specific cDNA library for detailed study of gene expression profile.

\section{Two-color transgenic zebrafish}

Recently, we have generated two-color transgenic zebrafish by injection of a mixture of DNA constructs, krt8-gfp and mylz2-rfp. In this transgenic line, GFP is expressed in skin cells while RFP is expressed in muscles. Consistent with the observation for mylz2-gfp transgenic lines described above, the transgenic line containing mylz2-rfp express RFP strongly and a red color is observed, indicating the feasibility to develop multiple transgenic color fish by using different color genes under different tissue-specific promoters.

\section{APPLICATIONS OF GFP TRANSGENIC ZEBRAFISH}

$g f p$ transgenic zebrafish has many applications in both basic research and biotechnology. In basic research in developmental biology, expression of a fluorescent protein gene under a tissue-specific promoter can be used to recapitulate gene expression program. This has been repeatedly demonstrated in both our studies and in the studies by others. Using $g f p$ transgenic zebrafish, it will be easier to trace cell lineage and cell migration. An excellent example is the gfp transgenic zebrafish generated under a heat shock inducible promoter and using this transgenic line certain neuronal cell lineage has been traced after activation of GFP expression by a laser beam. ${ }^{8)}$ In addition, $g f p$ transgenic zebrafish can be used for cell and nucleus transplantation experiments, as GFP expression and gfp transgene are good cellular and genetic markers for this type of experiments. It is particularly useful in nuclear transplantation if GFP expression is targeted to nucleus by addition of a nuclear localization signal to the transgenic DNA construct. Furthermore, GFP expression can be used to purify specific types of cells by fluorescence activated cell sorting (FACS). This is especially important if a specific type of cells is too small in number or too difficult to visualize and to isolate without a proper labeling. Finally, gfp transgenic zebrafish under tissue-specific promoters may serve as an excellent system in generation and characterization of mutants for the targeted tissues as the restricted expression of GFP may greatly facilitate the screening and analysis of mutants for the targeted tissue/organs.

In biotechnology, as we show here, gfp and $r f p$ transgenic fish may be used for generation of novel varieties of ornamental fish. Different color genes may be expressed in different tissues/organs by using different tissue-specific promoters. Currently, more mutant forms of $g f p$ genes for at least three more colors of fluorescent protein are commercially available: YFP for yellow fluorescent proteins, BFP for blue fluorescent protein and CFP for cyan fluorescent protein (Clontech). More colors can be obtained by crossing the fish of two different colors. For example, we have obtained organge fluorescent transgenic zebrafish by crossing the $g f p$ and $r f p$ transgenic fish.

To evaluate the ecological consequence of $g f p$ transgenic ornamental fish, the survival rates of transgenic fish were assessed from the offspring of crosses between mylz2-gfp fish and mylz2-rfp fish. In general, there is no apparent difference in survival rates among the four genotypes: wildtype, $g f p, \quad r f p$ and $g f p / r f p$. To evaluate reproduction success, a population of equal number of wild type and hemizygous $g f p$ transgenic fish was maintained for breeding for a week, the expected ratio (43.75\%) of transgenic offspring based on Mendelian genetics was observed $(43.60 \%, 1158$ out of 2656). Similar observation was also obtained for $r f p$ transgenic zebrafish. Thus, there is no indication that the fluorescent transgenic fish would have a reproductive advantage and pose a threat to the wild population. This is in contrast to the recently proposed Trojan gene hypothesis that growth hormone transgenic fish would be favourably selected in mating because of their larger body size and escape of such transgenic fish would cause eventual extinction of wild population. ${ }^{21)}$ 
Furthermore, fluorescent transgenic fish may be used for biomonitoring of environmental pollution. For example, $g f p$ or $r f p$ can be engineered under an inducible promoter and the expression of the transgene can be activated only under environmental stimulations such as by estrogenic compounds or by heavy metals. In such case, the fluorescent color will be an indicator of environmental pollution. Some feasible promoters for these purposes include those from vitellogenin, which is inducible by estrodiol, and $h s p 25$, which can be activated by heavy metals as well as by heat shock (our unpublished data). Currently we are introducing these promoter/color gene constructs into zebrafish for development of transgenic biomonitoring zebrafish.

\section{ACKNOWLEDGEMENTS}

Our work is supported by National University of Singapore, Ministry of Environment of Singapore, National Science and Technology Board (NSTB) of Singapore, and Public Utility Bureau of Singapore.

\section{REFERENCES}

1. Stuart GW, McMurray JV, Westerfield M. Replication, integration and stable germ-line transmission of foreign sequences injected into early zebrafish embryos. Development 1988; 103: 403-412.

2. Westerfield, M, Wegner, J, Jegalian, BG, DeRobertis, E.M., Pusche,l A.W. Specific activation of mammalian Hox promoters in mosaic transgenic zebrafish. Genes Dev. 1992; 6:591-598.

3. Amsterdam, A, Lin, S, Hopkins, N. The Aequorea victoria green fluorescent protein can be used as a reporter in live zebrafishembryos. Dev Biol. 1995; 171:123-129.

4. Moss, JB, Price, AL, Raz, E, Driever, W, Rosenthal, N. Green fluorescent protein marks skeletal muscle in murine cell lines and zebrafish. Gene 1996; 173:89-98.

5. Long Q, Meng A, Wang $H$, Jessen JR, Farrell MJ, Lin S. GATA-1 expression pattern can be recapitulated in living transgenic zebrafish using GFP reporter gene. Development $1997 ; 124$ : 4105-4111.

6. Higashijimas S, Okamoto H, Ueno N, Hotta Y, Eguchi G. High-frequency generation of transgenic zebrafish which reliably express GFP in whole muscles or the whole body by using promoters of zebrafish origin. Dev Biol 1997; 192: 289-299.

7. Higashijimas S, Hotta Y, Okamoto H. Visualization of cranial motor neurons in live transgenic zebrafish expressing green fluorescent protein under the control of the islet-1 promoter/enhancer. $J$ Neurosci $2000 ; 20$ : 206-218

8. Halloran MC, Sato-Maeda M, Warren JT, Su F, Lele Z, Krone PH, Kuwada JY, Shoji W. Laser-induced gene expression in specific cells of transgenic zebrafish. Development 2000; 127: 1953-1960.

9. Park, HC, Kim CH, Bae YK, Yeo SY, Kim SH, Hong SK, Shin J, Yoo KW, Hibi M, Hirano T, Miki N, Chitnis AB, Huh TL. Analysis of upstream elements in the $\mathrm{HuC}$ promoter leads to the establishment of transgenic zebrafish with fluorescent neurons. Dev. Biol. 2000; 227: 279-293.

10. Goldman D, Hankin M, Li Z, Dai X, Ding J. Transgenic zebrafish for studying nervous system development and regeneration. Transgenic Res. 2001; 10:21-33.

11. Kennedy BN, Vihtelic TS, Checkley L, Vaughan KT, Hyde DR. Isolation of a zebrafish rod opsin promoter to generate a transgenic zebrafish line expressing EGFP in rod photoreceptors. J Biol Chem. 2001; 276:1403714043.

12. Prasher DC, Eckenrode G, Ward WW, Prendergrast EG Cornmier MJ. Primary structure of the Aequorea victoria green-fluorescent protein. Gene 1992; 111: 229 233.

13. Gong Z, Yan T, Liao J, Lee SE, He J, Hew CL Rapid identification and isolation of zebrafish CDNA clones. Gene 1997; 201 : 87-98.

14. Gong $Z$. Zebrafish expressed sequence tags and their applications. Methods Cell Biol. 1999; 60: 213-233.

15. Liao J, Chan $\mathrm{CH}$, Gong $\mathrm{Z}$. An alternative linkermediated polymerase chain reaction method using a dideoxynucleotide to reduce amplification background. Anal Biochem 1997; 253: 137-139.

16. Matz MV, Fradkov AF, Labas YA, Savitsky AP, Zaraisky AG, Markelov ML, Lukyanov SA Fluorescent proteins from nonbioluminescent Anthozoa species. Nature Biotech 1999; 17: 969-973.

17. Ju B, Xu Y, He J, Liao J, Yan T, Hew CL, Lam TJ, Gong Z. Faithful expression of Green Fluorescent Protein (GFP) in transgenic zebrafish embryos under control of zebrafish gene promoters. Dev Genet 1999; 25: $158-167$.

18. Xu Y, He J, Tian HL, Chan CH, Liao J, Yan T, Lam TJ. Gong $Z$. Fast skeletal muscle-specific expression of a zebrafish myosin light chain 2 gene and characterization of its promoter by direct injection into skeletal muscle. DNA Cell Biol 1999; 18: 85-95.

19. Xu Y, He J, Wang $X$, Lim TM, Gong $Z$ Asynchronous activation of 10 muscle-specific protein (MSP) genes during zebrafish somitogenesis. Dev Dyn 2000; 219: 201-215.

20. Devoto SH, Melancon E, Eisen JS, Westerfield, M. Identification of separate slow and fast muscle precursor cells in vivo, prior to somite formation. Development 1996; 122:3371-3380

21. Muir WM, Horward RD. Possible ecological risks of transgenic organism release when transgenes affect mating success: sexual selection and the Trojan gene hypothesis. Proc. Natl. Acad. Sci. USA 1999; 96: 13853-13856 